\title{
Experimental study on dynamic characteristics of UHPC pedestrian cable stayed bridge
}

\author{
W. J. Chin, Y. J. Kim, E. S. Choi, J. Y. Kang \& B. S. Kim \\ Structural Engineering \& Bridge Research Division, \\ Infrastructure Research Department, \\ Korea Institute of Construction Technology, Republic of Korea
}

\begin{abstract}
The first pedestrian cable stayed bridge using ultra-high performance concrete (UHPC) has been designed and constructed in Korea. The 200MPa class UHPC is the fruit of the research performed by the Korea Institute of Construction Technology (KICT). UHPC is an innovative and ultra-high strength material with compressive strength larger than $180 \mathrm{MPa}$ that has been developed to secure the ductility and strength of the structural members. UHPC exhibits significantly improved tensile, flexural and shear strengths enabling to minimize the crosssectional depth. However, the significant autogenous shrinkage developed by UHPC is also potentially favouring the occurrence of initial cracking in the case of structures presenting complicated shapes. Considering such characteristics of UHPC, a pedestrian cable stayed bridge using UHPC has been erected inside the site of KICT. The dynamic characteristics of the precast UHPC deck were evaluated through performance tests on the first UHPC pedestrian cable-stayed bridge. Examination of the maximum acceleration and displacement responses at the ends of the girder is carried out according to the excitation method. The estimated natural frequency, and measured vertical displacements and accelerations are compared with the design criteria. The corresponding results verify the applicability of UHPC not only to pedestrian cable stayed bridge but also to roadway cable stayed bridge.
\end{abstract}

Keywords: ultra high performance concrete, pedestrian cable stayed bridge, dynamic characteristics, hybrid concept. 


\section{Introduction}

Ultra high performance concrete (UHPC) is a material that has been developed firstly in France by Ductal ${ }^{\circledR}$ to overcome the drawbacks encountered in existing concretes by providing enhanced strength through the introduction of steel fibers and admixtures. UHPC secures the ductility and strength of the structural members by significantly improving the tensile, flexural and shear strengths as well as the resistance to cracking and impact. Owing to such properties, the Korea Institute of Construction Technology (KICT) also developed the KICTUHPC through an in-house research program. KICT-UHPC is a mix composed of a fiber-reinforced cement compound featuring a design compressive strength exceeding $150 \mathrm{MPa}$ and design splitting tensile strength beyond $5 \mathrm{MPa}$, a waterto-binder ratio lower than 0.24 and a volumetric percentage of reinforcing fibers larger than $2 \%[1-3]$.

There are very few applications of UHPC for bridge structures. In Korea, a typical example is the Seonyu pedestrian bridge erected in 2002 using French technology. In foreign countries, the Sherbrooke pedestrian bridge built in 1997 in Canada has pioneered the application of UHPC in bridges. This example was followed by the Shepherds Bridge in Australia, the Papatoetoe station viaduct in New Zealand, the Chabotte Bridge in France and the Sakata Mirai pedestrian bridge in Japan. Currently, KICT is conducting a project called "SUPER BRIDGE 200 - Development of Low Cost and Long Life Hybrid Cable Stayed Bridge". This project aims to reduce the construction and maintenance costs of long-span bridges by $20 \%$ and double their lifetime through the exploitation of UHPC.

A survey revealed that the current technological level of Korea in the field of long-span bridges is reaching about $73 \%$ that of advanced countries. In spite of the numerous long-span bridges erected or under construction in the Korean peninsula, it is noteworthy that the key technologies adopted for the construction of most of these bridges were relying on those provided by advanced countries like USA, Europe or Japan. This stresses the very poor self-reliance of our domestic technology and the urgency of securing original technologies.

A previous study examined and compared the economic efficiency of the design of a hybrid cable stayed bridge using UHPC for the site of Seohae Bridge, a cable stayed bridge currently in operation in the western coast of Korea. The investigation found that the weight of the superstructure could be lightened by $22 \%$ through the hybrid cable stayed bridge using UHPC. The study also verified that the construction cost of the superstructure was reduced by $25 \%$. The comparative analysis of the economic efficiency of the hybrid cable stayed bridge extended to span lengths of 200, 400 and $600 \mathrm{~m}$ showing that the construction cost reduced respectively by 15,28 and 24\%. Accordingly, our research team implemented the planning, design, curing, placing and erection of a full scale pedestrian cable stayed bridge using the developed UHPC inside the site of KICT. The bridge is a one-pylon pedestrian cable stayed bridge with its fan type cables arranged in 3 plans linking the main building and the third floor of the newly built laboratory building of KICT. The bridge features a structure 
resisting the live loads through the stiffness of the pylon and maintaining equilibrium through the permanent load of the girder. This bridge has particular meaning since it is the first pedestrian cable stayed bridge applying UHPC and also because it adopts the hybrid concept. The hybrid concept stands for the optimized exploitation of materials that is, UHPC is applied for the deck and steel is used for the upper part of the pylon to anchor the cables while ordinary concrete is adopted for the end slab in order to achieve balance of the forces. This pedestrian bridge constitutes a unique and creative initiative, which has been realized by our own technology and provides a test bed demonstrating that the economically efficient construction of long-span bridges can be achieved through the use of UHPC. Our research team verified the promising feasibility and applicability of UHPC cable stayed bridges through the erection of this pedestrian cable stayed bridge [1-4].

Besides, the natural frequencies, vibrational acceleration and dynamic displacement of the UHPC cable stayed bridge were measured during a preliminary dynamic test. These responses were used to evaluate the dynamic characteristics of the bridge. In addition, as the pedestrian cable stayed bridge was a cantilevered structure, vibrations exceeding the vertical vibrational criteria were observed. Accordingly, a vibration control system using vertical vibration control device was also proposed [2].

\section{Construction of the UHPC pedestrian cable stayed bridge}

\subsection{Summary of design}

The ordinary section of the upper girder was planned as a precast girder using UHPC with compressive strength of $180 \mathrm{MPa}$. The segments of the precast girder were connected using $20 \mathrm{~mm}$ steel bars after having been epoxy-bonded. The section contiguous to the pylon was planned to be cast-in-place concrete considering the connection with the pylon. Edge girder was selected for the cross-section of the upper girder in order to develop the compressive performance of UHPC at the most and the depth of the deck was minimized. PHC piles were applied. The SDA method using steel pipe casing was chosen for the pile foundation to prevent the collapse of the wall of the boring since the bed rock stood at a depth of $24.5 \mathrm{~m}$. This choice was also governed owing to the small vibration and noise produced by the method considering the neighbouring buildings. Anchor plates were welded at the top of the pylon to fix the cables. The joint between steel and concrete was conceived as a monolithic structure by installing shear keys at the bottom plate and welding the reinforcing bars of the concrete section to the plate. High strength concrete of $40 \mathrm{MPa}$ was applied for the upper part of the pylon and ordinary concrete of $24 \mathrm{MPa}$ for the bottom of the girder. OSS cable system (Pfeifer) was adopted owing to its appropriateness for a small scale pedestrian bridge. The cables exhibited a diameter of $20.1 \mathrm{~mm}$ and allowable stress of 1,500 $\mathrm{MPa}$. 


\subsection{Overview of the construction}

The conceptual drawing of the UHPC pedestrian cable stayed bridge is illustrated in Figure 1. Figures 2 and 3 present the erection of the precast deck and the completed Super Bridge 1.

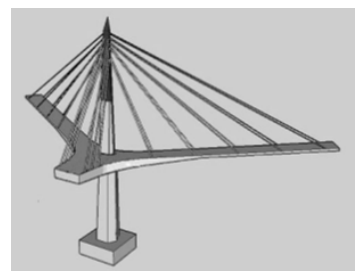

Figure 1: Conceptual drawing of super bridge 1.

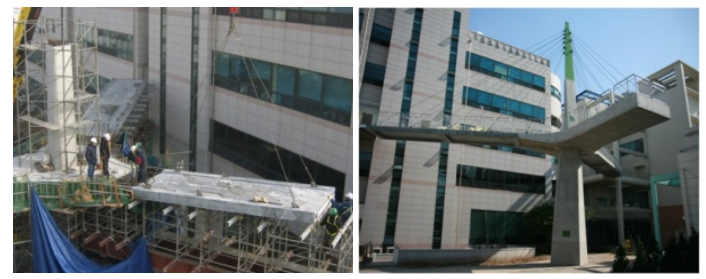

Figure2: Erection of the Figure3: Erection of UHPC deck. super bridge 1 .

\section{Preliminary vibration test of the UHPC footbridge}

\subsection{Summary of dynamic characteristics test}

Recently, bridge structures are exhibiting reduced size of their members through the increasing use of high strength materials and steel, and gradually discarding non-structural members when a wider space is needed according to the purpose of the structure. However, these features result unfortunately in a significant reduction of the damping effect. Particularly, in the case of long-span pedestrian bridges, which are characterized by a low natural frequency, excessive vibrations are likely to occur under dynamic loads such as the walking loads of the pedestrians. Such undesirable vibrations are not only causing discomfort to the users but may also degrade the safety of the structure. These vibrations of the bridge caused by walking loads affect the structure itself as well as the pedestrians crossing the bridge in diverse forms. Apart from the discomfort of the pedestrians, this phenomenon may provoke vibrations with amplitude larger than the limiting criteria and cause problems in terms of the serviceability and safety of the bridge when the frequency of the walking load synchronizes with the natural frequency of the structure. Moreover, the walking load presents various load-time history models according to diverse factors like the weight of the pedestrian, the frequency and speed of walking, the type of walking and individual walking habits. The vibrational phenomenon due to such walking load constitutes a load source sufficient to provoke dynamic behavioural responses of the bridge but research dedicated to the dynamic behavioural characteristics of bridge structures has been rather neglected in Korea and overseas to date. This 
explains why Korea has not still provided established design specifications nor implemented systematic studies on this subject $[5,6]$.

This study evaluates the vibrational serviceability of the UHPC pedestrian cable stayed bridge through comparative analysis using both analytic and experimental methods so as to provide appropriate countermeasures. Therefore, a three-dimensional model of the bridge was established and dynamic analysis was performed using time histories of the walking load. Dynamic test was carried out to evaluate the dynamic behaviour of the structure according to major factors determining the walking load like the walking weight and frequency. This enabled to verify the mode shapes of the bridge and estimate its vibrational serviceability. The maximum values of the vibrational acceleration and natural frequency as well as the dynamic deflection at the end of the deck were measured through forced vibration test. The natural frequency of the bridge structure can be estimated by measuring the dynamic responses under forced vibrations within the elastic range of the structure. In general, responses can be obtained clearly when load with relatively large energy is applied to the structure, but applying loads on the real structure is delicate. A recommended method is to estimate the natural frequency by evaluating the frequencies provoked by the resonance occurring after having applied the load by adjusting the rpm of a small scaled exciting device. However, the exciting device needs to be anchored to the bridge. Therefore, this method was discarded and another method was adopted. Figures 4 and 5, 6 present the UHPC test bed and installed sensors. The natural frequency was thus estimated by measuring the acceleration occurring when loaded by a shock wave produced by small hits given by a hammer (Figure 7). In addition, the natural frequency was estimated under loading conditions corresponding to walking or jumping of pedestrians since the structure is a pedestrian bridge (Figure 8). Figure 9 shows a picture of the dynamic test.

\subsection{Dynamic characteristics test by impact loading}

As the result of the natural frequency estimated from the hitting test by hammer, Figure 10 plots the time history of the acceleration measured at the end of the upper girder of the pedestrian bridge, and Figures 11 and 12 present the FFT analysis results. As can be seen in Figure 13, dynamic displacement did practically not occur with a value of about $0.05 \mathrm{~mm}$.

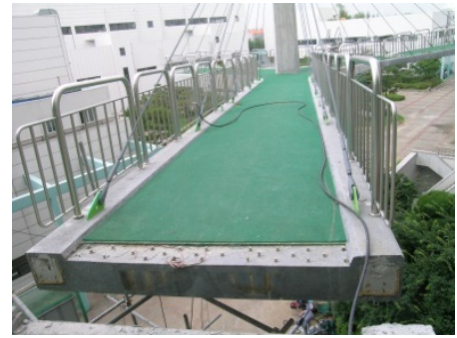

Figure 4: $\quad$ UHPC footbridge.

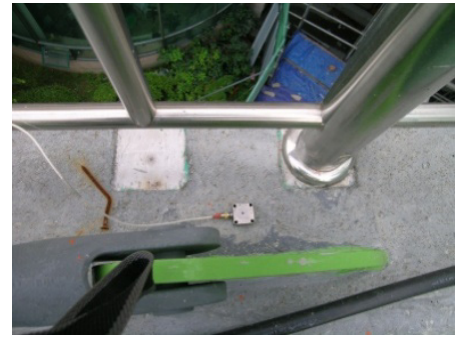

Figure 5: Accelerometer. 


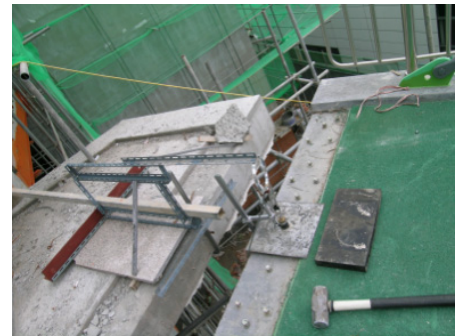

Figure 6: Accelerometer hammer.

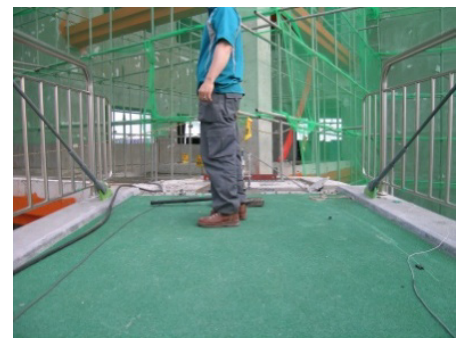

Figure 8: Vibration test by.

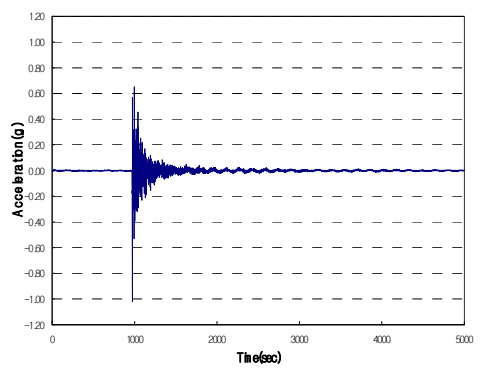

Figure 10: Acceleration time history (hammer).

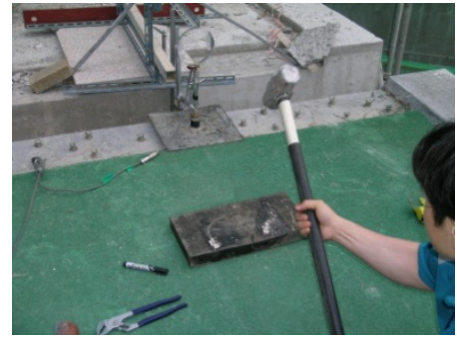

Figure 7: Vibration test using impact.

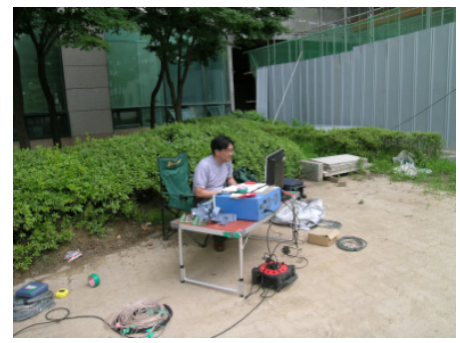

Figure 9: Dynamic test pedestrian impact

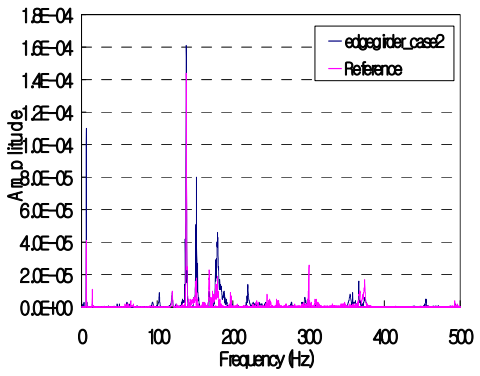

Figure 11: $\quad$ PSD $(500 \mathrm{~Hz})$.

Figures 14 to 17 show the results of the loading test by jumping of pedestrian. It can be seen in Figure 11 presenting the analysis result of the test using hammer that the energy is concentrated in the higher frequencies. Besides, the FFT analysis results of the test by jumping of pedestrian reveal that the energy is concentrated in the lower frequencies. Accordingly, it appears that the vibration 


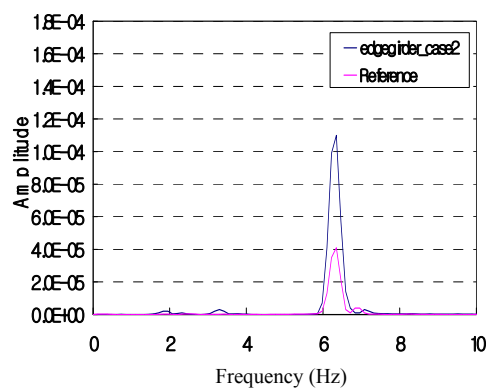

Figure 12: $\quad$ PSD $(10 \mathrm{~Hz})$.

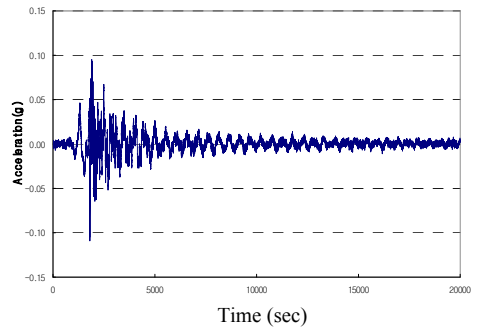

Figure 14: Acceleration time history (jumping of pedestrian).

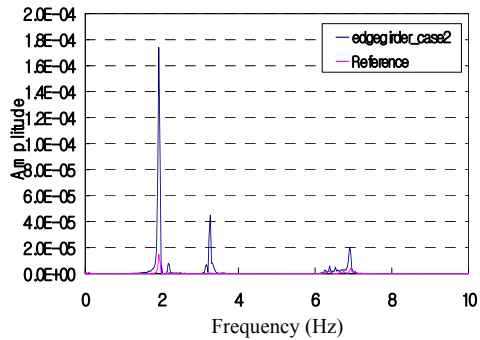

Figure 16: $\quad$ PSD $(10 \mathrm{~Hz})$.

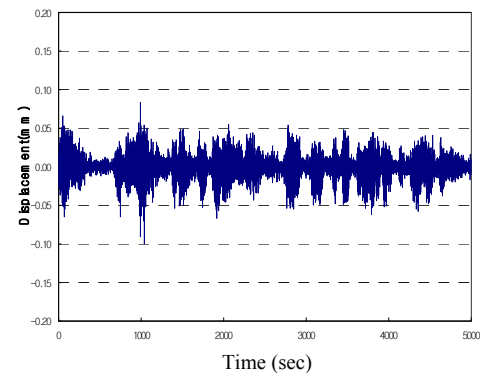

Figure 13: Displacement time history (hammer).

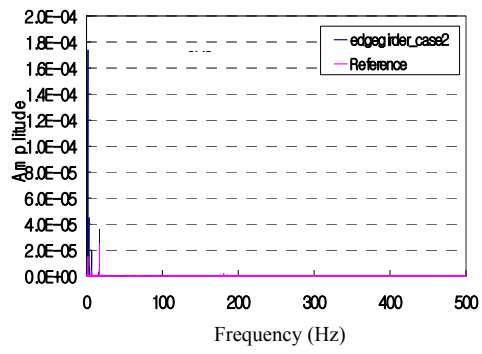

Figure 15: $\quad$ PSD $(500 \mathrm{~Hz})$.

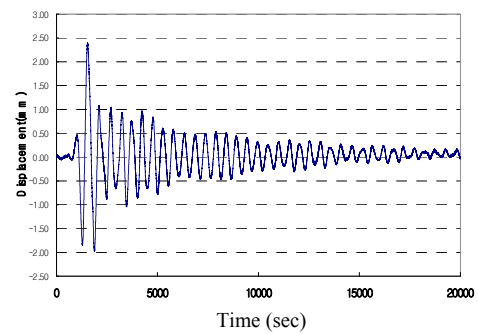

Figure 17: Displacement time history (jumping of pedestrian).

test by jumping of pedestrian constitutes an adequate testing method for estimating the natural frequency of the UHPC pedestrian cable stayed bridge.

Structural analysis was conducted using the software SAP2000 during the design of the pedestrian bridge. The results of the analysis indicated that the 
natural frequency of the UHPC pedestrian cable stayed bridge was $2.1 \mathrm{~Hz}$. In comparison, the natural frequency estimated from the pedestrian jumping test was $1.92 \mathrm{~Hz}$. For the maximum acceleration, the jumping test provided a value of about $0.11 \mathrm{~g}$ and the corresponding maximum dynamic deflection was $2.4 \mathrm{~mm}$.

\section{Dynamic characteristics test by pedestrian load}

Dynamic test by pedestrian walking and jumping was conducted for the UHPC pedestrian cable stayed bridge. The testing method is summarized in Table 1 and Figures 18 and 19 show pictures of the test. Tables 2 and 3 arrange the experimental results. It can be seen that an identical natural frequency of $1.91 \mathrm{~Hz}$ was estimated during the running of two pedestrians. Since an exciting load with frequency of $2 \mathrm{~Hz}$ is applied during the walking of pedestrian, the installation of TMD is recommended to prevent the occurrence of resonance. Note that TMD was also installed in the Seonyu Bridge, the first UHPC footbridge in Korea [79].

Table 1: Test methods for performance check.

\begin{tabular}{|c|c|c|}
\hline \multicolumn{2}{|c|}{ Case } & Testing method \\
\hline \multirow{4}{*}{ Toward main building 1} & 1 & 1 pedestrian - jumping \\
\cline { 2 - 3 } & 2 & 1 pedestrian - walking \\
\cline { 2 - 3 } & 3 & 2 pedestrians - walking \\
\cline { 2 - 3 } & 4 & 2 pedestrians - running \\
\cline { 2 - 3 } & 5 & $\begin{array}{c}\text { 1 pedestrian - jumping at TMD } \\
\text { installing position }\end{array}$ \\
\hline \multirow{2}{*}{ Toward main building 2 } & 1 & 1 pedestrian - jumping \\
\cline { 2 - 3 } & 2 & 1 pedestrian - jumping (heavy weight) \\
\hline
\end{tabular}

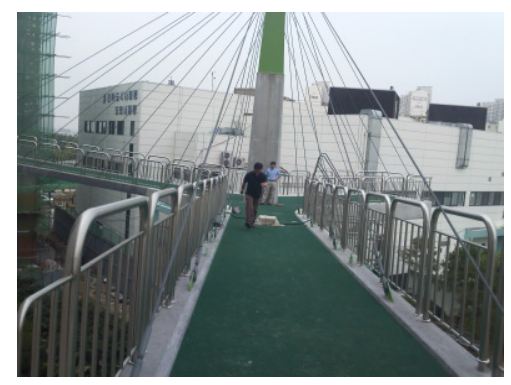

Figure 18: Vibration

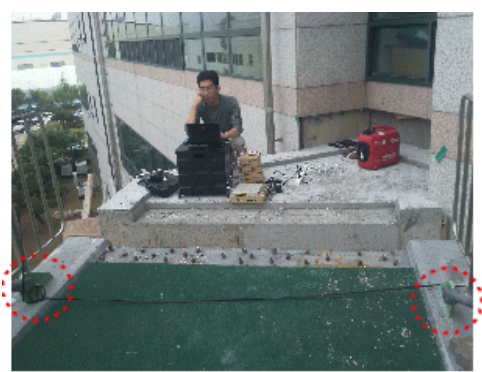

test Figure 19: Field measurement test. (pedestrian walking). 
Table 2: $\quad$ Results of vibration test (toward main building 1).

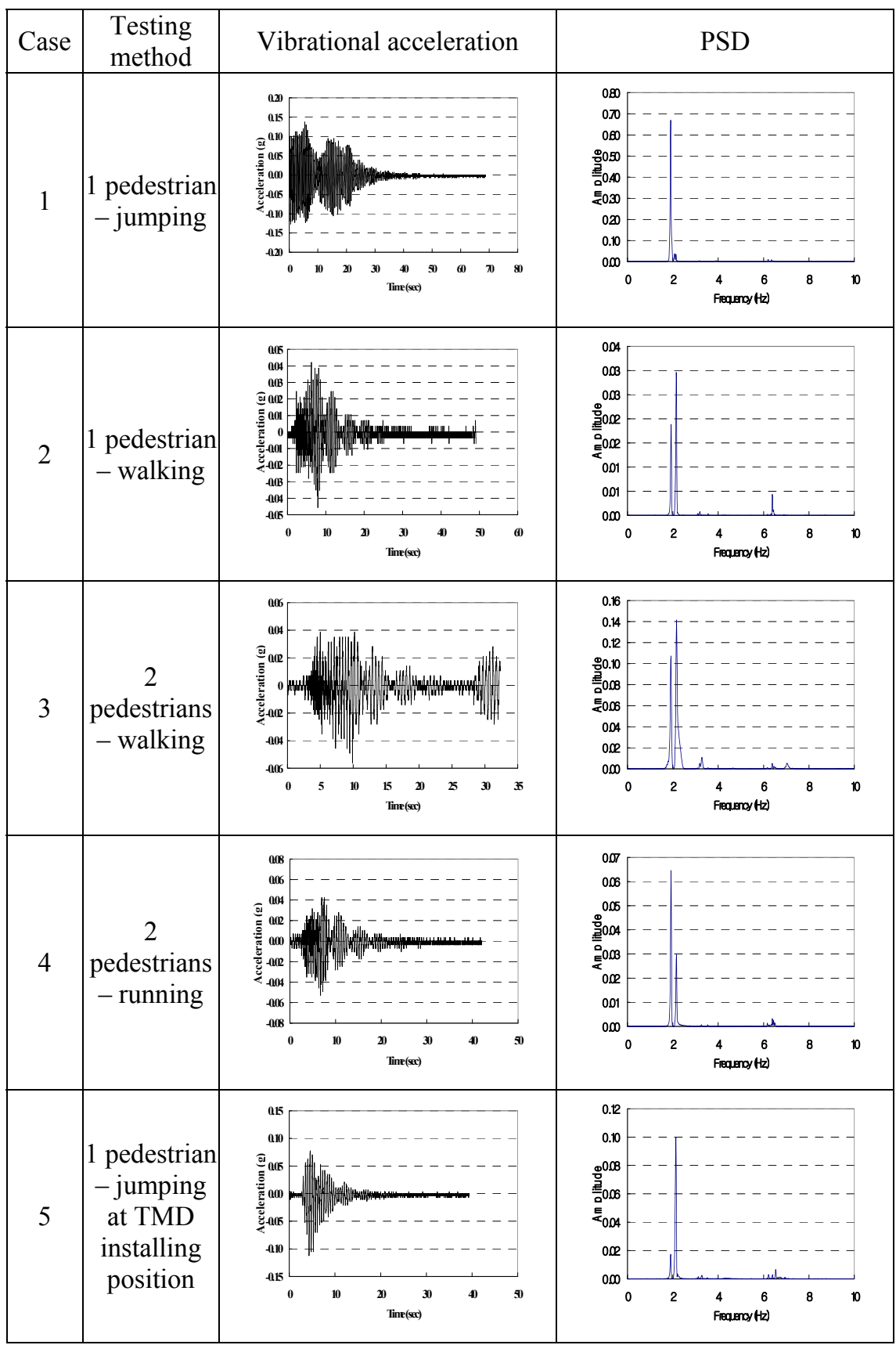


Table 3: $\quad$ Results of vibration test (toward main building 2).

\begin{tabular}{|c|c|c|c|}
\hline Case & $\begin{array}{l}\text { Testing } \\
\text { method }\end{array}$ & Vibrational acceleration & PSD \\
\hline 1 & $\begin{array}{c}1 \text { pedestrian } \\
- \text { jumping }\end{array}$ & 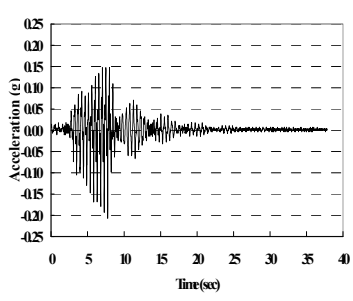 & 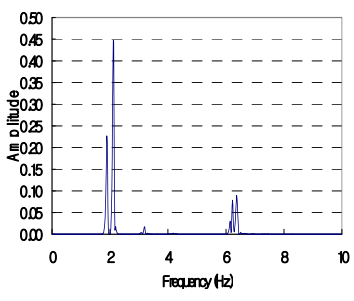 \\
\hline 2 & $\begin{array}{c}1 \text { pedestrian } \\
\text { - jumping } \\
\text { (heavy } \\
\text { weight) }\end{array}$ & 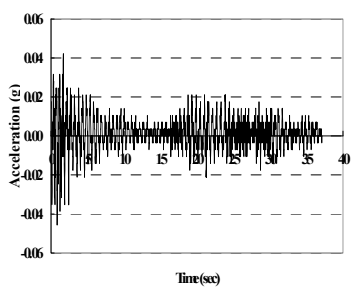 & 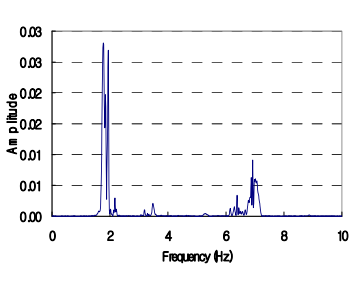 \\
\hline
\end{tabular}

\section{Conclusions}

A full-scale pedestrian cable stayed bridge reflecting sufficiently the characteristics of the KICT-UHPC has been planned, designed, cured, placed and erected inside the site of the Korea Institute of Construction Technology (KICT). This footbridge is the first pedestrian cable stayed bridge applying UHPC and adopts the hybrid concept, which aims the efficient exploitation of materials. Our research team verified the practicability and feasibility of UHPC pedestrian cable stayed bridge by means of the actual erection of this test bed linking buildings 1 and 2 inside the site of KICT. In addition, a preliminary vibration test evaluated the dynamic characteristics of the footbridge through measurement of the natural frequency, vibrational acceleration and dynamic deflection of the structure. The results indicated that satisfactory serviceability in terms of vibration was secured for most of the pedestrian walking load cases. However, responses exceeding the limiting criteria occurred in the case of impacts induced by the jumping of pedestrian. Structural analysis was conducted using the software SAP2000 during the design of the pedestrian bridge. The results of the analysis indicated that the natural frequency of the UHPC pedestrian cable stayed bridge was 2.1 Hz. In comparison, the natural frequency estimated from the pedestrian jumping test was $1.92 \mathrm{~Hz}$. For the maximum acceleration, the jumping test provided a value of about $0.11 \mathrm{~g}$ and the corresponding maximum dynamic deflection was $2.4 \mathrm{~mm}$. Since an exciting load with frequency of $2 \mathrm{~Hz}$ is applied during the walking of pedestrian, the installation of TMD (Tuned Mass Damper) is recommended to prevent the occurrence of resonance. Further studies are 
required to apply diversified test variables related to the vibration test of the footbridge and for the evaluation of the vibrational characteristics of the UHPC pedestrian cable stayed bridge like the maximum acceleration, dynamic deflection and modal shapes through various vibration tests and analyses.

\section{Acknowledgements}

This study was carried out as part of the "R\&D on Design and Construction System of Hybrid Cable Stayed Bridges" at the Korea Institute of Construction Technology. The authors express their gratitude for the support.

\section{References}

[1] Korea Institute of Construction Technology (KICT), Development of integrated technology for hybrid cable stayed bridge, Research report, 2009.

[2] Chin, W.J. et al, Evaluation of the dynamic characteristics of UHPC pedestrian cable stayed bridge (Super Bridge 1), Proc. of KSCE Annual Confer., 2009.

[3] Ministry of Construction and Transportation, Development of bridge girder using ultra high performance cementitious composite materials, Report of Construction Core Technology R\&D Program, 2003.

[4] Kim, W.J., Super Bridge 200: the ambition of bridge in Korea, KICTzine, May/June volume, 2008.

[5] Korea Infrastructure Safety and Technology Corporation (KISTEC), Establishment of evaluation procedure for the vibration and deflection of bridges in terms of serviceability, Final report of the Construction Innovation Program, 2000.

[6] Cho, I.W., A study on the vibrational serviceability of cable supported footbridge considering walking loads, Master thesis at Yonsei University, 2005.

[7] K-enc Research Institute, Report of the installation of TMD (EC-FTMD) (UHPC pedestrian cable stayed bridge at KICT), 2009.

[8] Hartley et al, Investigation of pedestrian walking loads on a cable stayed footbridge using modal testing and FE model updating, Proc. of IMAC-XVII, 1999.

[9] Lee, J.I. et al, Efficient vibration control of footbridge using TMD, Proc. of KSCE Annual Confer., 2002. 